\title{
Online bearing lubricant sensing by mode conversion of surface acoustic waves
}

\author{
Gerhard Lindner, Christoph Brückner, Martin Schmitt \\ Institute of Sensor and Actuator Technology, Coburg University of Applied Sciences, \\ Am Hofbräuhaus 1 \\ 96450 Coburg, Germany \\ lindner@hs-coburg.de
}

\begin{abstract}
A novel approach to online condition monitoring of the lubricant status in bearings is introduced, which is based on the mode conversion effect of surface acoustic waves: The excitation of Lamb waves on an outer surface of a bearing by attached piezoelectric interdigital transducers results in an interaction of these Lamb waves with the lubricant at the inner surface. This interaction influences the transmission of the Lamb waves to a second transducer due to the mode conversion effect of surface acoustic waves in contact with a liquid. The presence of the lubricant causes a loss of acoustic energy from the Lamb waves by a compression sound wave radiated into the liquid. Therefore, both the amplitude and the transmission time of such leaky Lamb waves depend on the presence and the status of the lubricant within the bearing. This concept has been tested with different types of bearings - axial cylinder roller bearings, radial ball bearings and radial cylinder roller bearings - at different rotation conditions and with oil and grease lubricants. In all cases an influence of the amount of lubricant both on the amplitude and the transmission time of the acoustic signal has been observed. In addition, a modulation of signal amplitude and transmission time was registered, which was correlated with the rotational movement of the rolling elements within the bearing. The comparison of the modulation frequency with the rotational frequency allows a determination of the motion and the slip of the bearing cage.
\end{abstract}

\section{Introduction}

Inadequate lubrication of bearings may result in a failure of the whole bearing and consequently severe damages of engines, in which that bearing is operating. Therefore, there is a demand for sensing concepts allowing an online surveillance of the lubricant state during the operation of the bearing. The measurement of the rise of temperature of the bearing - either at the outer shell or near the rotating axis - is a common indication of a malfunction of the bearing, but this approach may not be sufficient for the prevention of damage from strongly loaded bearings. More detailed information about the lubricant state within the bearings have been obtained by acoustic methods, in particular with ultrasonic waves launched into the bearing structure [1,2]. Taking advantage of specific properties of selected modes of surface acoustic waves, in particular Lamb waves or waves in the transition regime between Lamb and Rayleigh waves (in the following called "Lamb-like" waves), a rather strong sensitivity of the propagation parameters of such waves with respect to the presence and the status of the lubricant within the bearing could be achieved [3].

The key issue in the utilization of this particular mode of surface acoustic waves is the possibility of a spatial separation of the excitation of and the sensing with such waves: Since those waves typically propagate on plates, the displacements on both surfaces of a plate are elastically coupled - i.e. whereas the wave may be excited at the outer surface of the outer shell of a bearing, the corresponding displacements on the inner surface of the outer shell, which is in contact with the lubricant, may be influenced by interactions with the lubricant, which again affects the propagation properties of the wave. Of particular interest in that context is the mode conversion effect of surface acoustic waves, which results in a substantial loss of acoustic power due to diffraction, if the surface of wave propagation is in contact with a liquid, whose velocity of sound is smaller than the propagation velocity of the surface acoustic wave. [4, 5].Correspondingly, the presence of a lubricant will result in a reduction of the amplitude of a surface acoustic wave of this type travelling along the outer shell of a bearing and in some change of the propagation velocity. 
In this contribution it is demonstrated that this concept can be applied with Lamb-like waves to different types of bearings - e.g. axial cylinder roller bearings, radial ball bearings and radial cylinder roller bearings - with different lubricants. Results of measurements of both the amplitude and the transmission time of short wave pulses as a function of the amount of lubricant in the bearing at different rotational speeds are shown, which additionally allow a determination of the slip of the bearing cage. Such measurements have been performed at unloaded bearings up to now; the results obtained so far, however, encourage for an extension of the investigations to loaded bearings in the future.

\section{Experimental set-up and measurement methods}

The following bearings have been used for the investigations reported herein: Radial ball bearings (type 6305 and 6306) and radial cylinder roller bearings (type N305-E-TVP2 and N306-E-TVP2). The bearings were coupled with an electrical motor and thus could be driven at different rotational speeds. They could be filled with specific amounts of different lubricants (oil or grease). Transmission pathways for Lamb-like waves were established by piezoelectric ceramic transducers made of PZT with extensions of $6 \times 4 \times 1$ $\mathrm{mm}$ attached to the outer surface of the bearings with an epoxy glue. The electrode at the bottom surface of the transducer forms a two-finger structure with a pitch of about $3 \mathrm{~mm}$, which allows the excitation of the antisymmetric $\mathrm{A} 0$ mode of a Lamb wave on the outer shell of the bearing at an excitation frequency of $1 \mathrm{MHz}$. The coupling of the vibrations of both the outer and the inner surface of the outer shell in this acoustic wave mode results in an acoustic radiation loss at the interface with a lubricant as indicated by fig. 1.

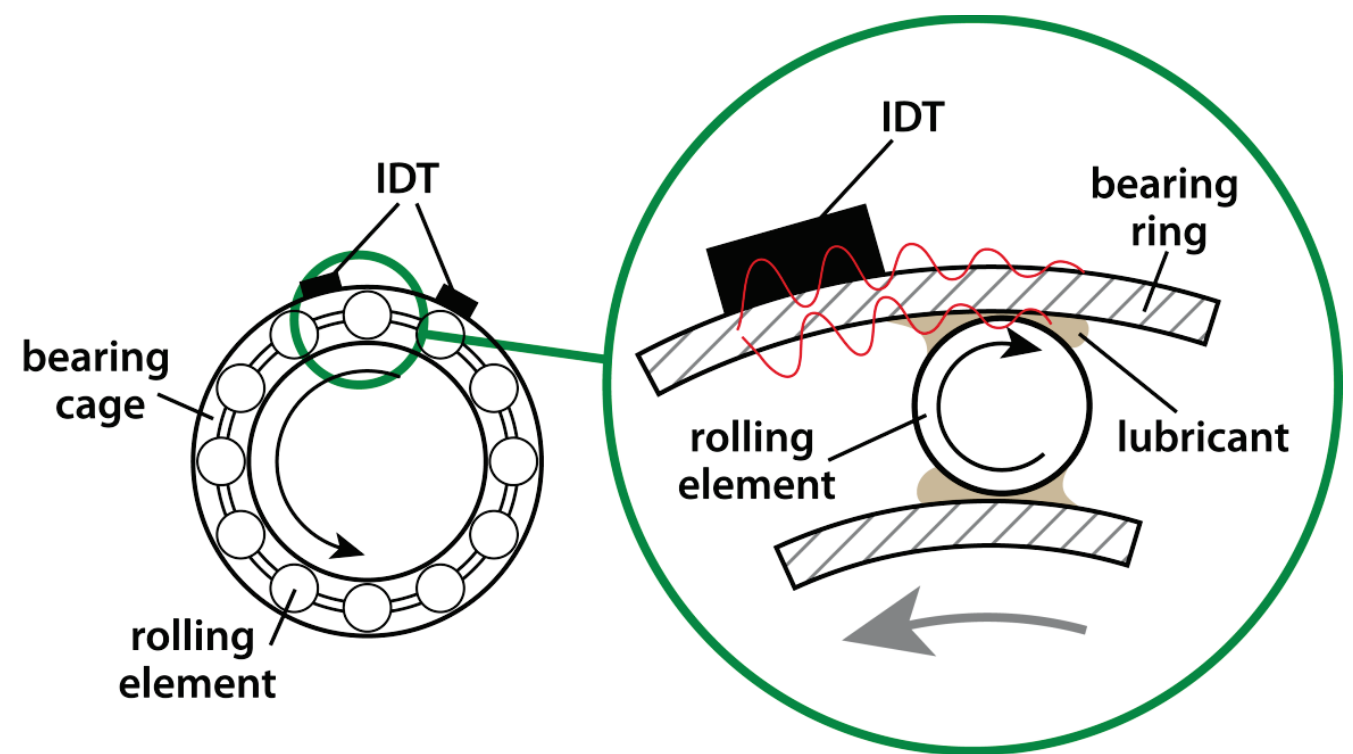

Fig 1: The surface acoustic wave is propagating on the static bearing ring and is influenced by the interaction of the amount of oil and the rolling elements.

The measurements of the wave propagation were performed in the pulsed mode, i.e. one of the transducers was excited with a short sine burst and the signals of the transmitted wave pulses produced by the second transducer were recorded by a digital storage oscilloscope and analyzed with respect to transmission time and maximum amplitude. The repetition rate of the pulsing was $4 \mathrm{kHz}$ and thus much larger than the rotational frequency of the bearings. The transmission times have been extracted from the zero crossing of the receiver signal immediately preceding the maximum amplitude. Since the transducers may emit and receive surface acoustic wave pulses into or from two opposite directions, respectively, always pairs of wave pulses are travelling along the outer shells. In order to be able to discriminate between the two different pathways with a pair of wave pulses, the receiver transducer is located at a non-symmetrical angular position with respect to the emitter transducer (fig. 1). Then the receiver signals obtained from the arrival of the two wave pulses travelling in opposite directions are well separated in time and can easily be distinguished. This allows an internal compensation of external effects affecting both wave pulses in the same way such as external temperature or changes in the excitation amplitudes. 
A careful analysis of the receiver signal during the revolution of the bearing revealed small periodic changes of the signal parameters as shown in fig. 2, which turned out to be periodic in time (fig. 3 ). The frequency of this modulation was strongly correlated with the rotational frequency of the bearing and the number of rolling elements within the bearing. Therefore it was concluded that information about the movement of the rolling elements and the cage connecting those elements could be obtained from that modulation. In summary, the following relationships between signal parameters of the acoustic wave pulses and bearing conditions were investigated by the experiments reported herein at different bearing types: (i) influence of the amount of lubricant - oil or grease - on the signal parameters at a certain rotational speed and (ii) variation of signal parameters in the course of time due to the movement of the balls or cylinders inside the bearing at different rotational speeds.

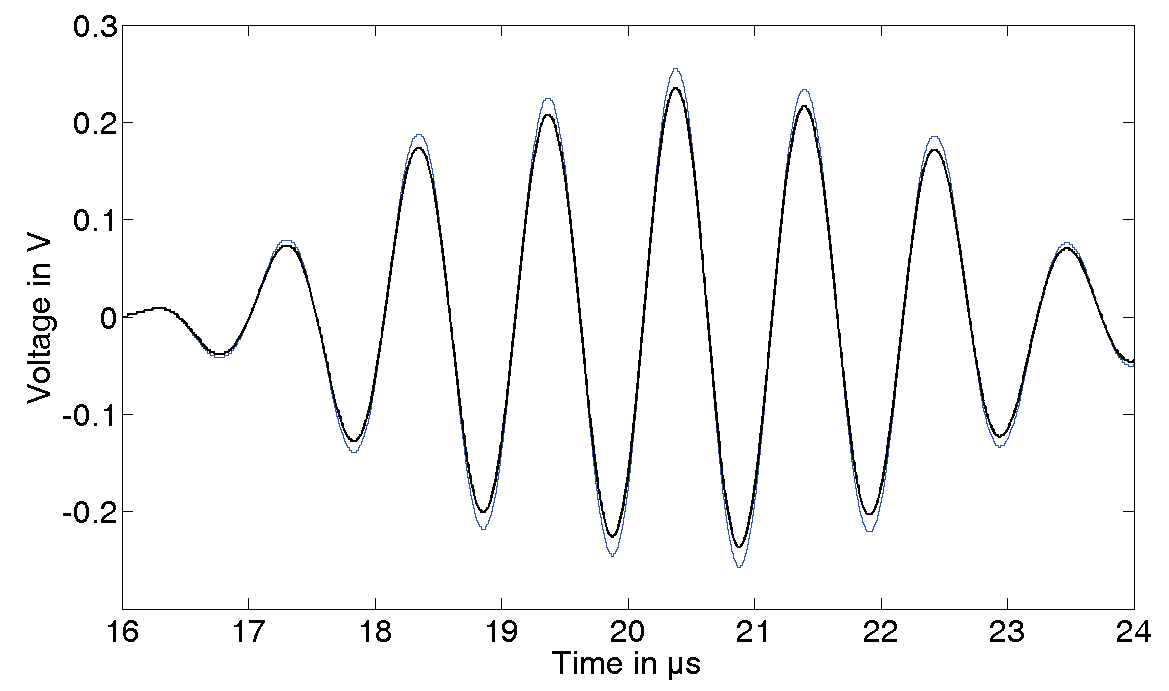

Fig. 2: Receiver signals measured at the time of amplitude maxima (blue) and minima (black) during the rotation of the bearing
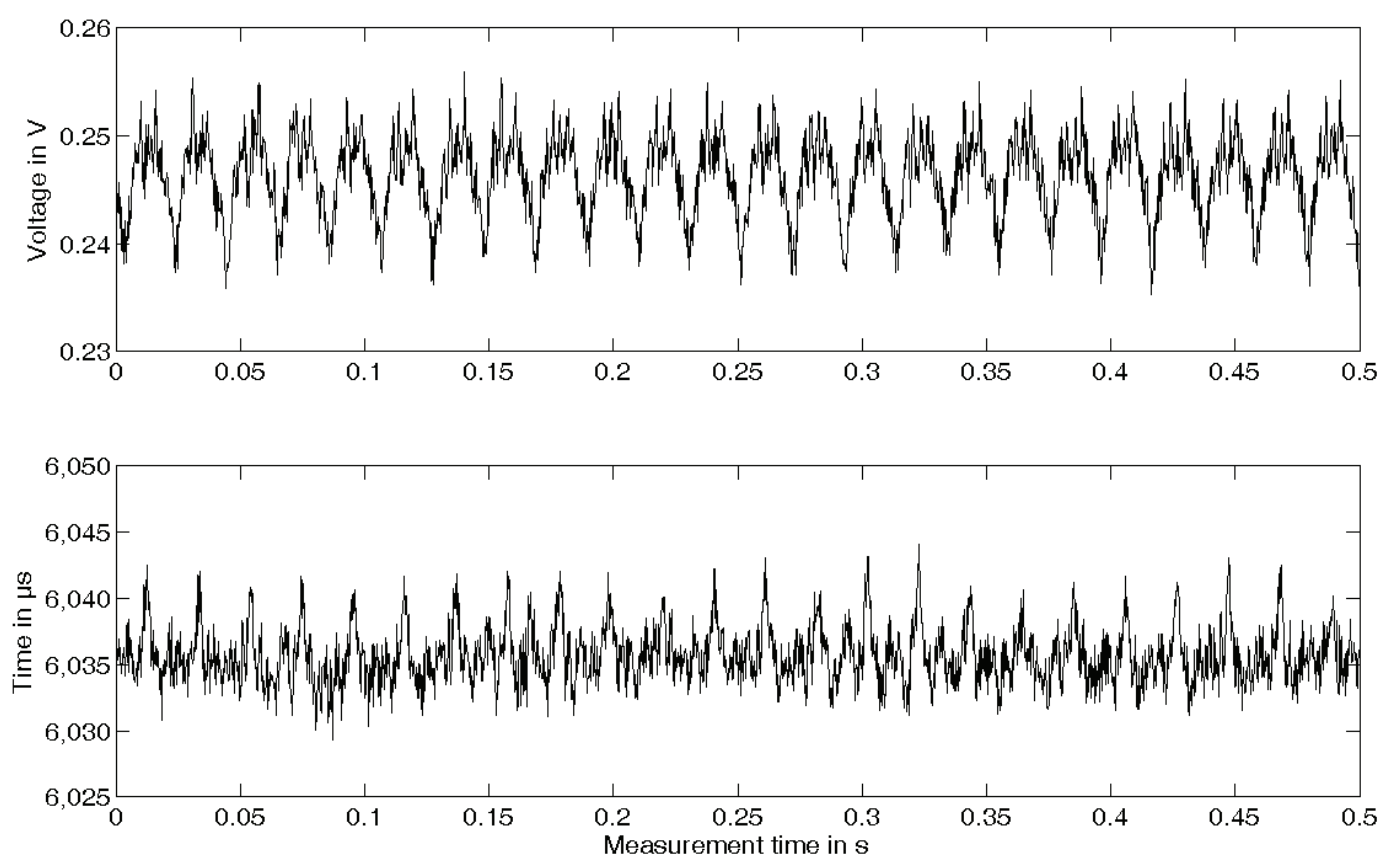

Fig.3: Modulation of amplitudes and transmission times of the receiver signal at a rotational speed of 320 rpm 


\section{Results}

\subsection{Amount of Lubricant}

The influence of the amount of lubricant on the signal parameters was investigated with the cylinder roller bearings filled with oil and with the ball roller bearings filled with grease. Both changes of the baseline values of the signal parameters as well as of the modulation amplitudes at a certain rotational speed have been recorded. Although the modulation of the signal parameters turned out to be a periodic and not a stochastic phenomenon, the resulting standard deviations of the measured parameters during one measurement run were calculated from the data for simplicity, which predominantly reflects the respective amplitudes of modulation. With oil lubricant, an almost monotonous decrease of the amplitude and increase of the transmission time with the amount of lubricant was observed, whereas the almost monotonous increase of the standard deviations of both parameters indicate a growth in the modulation amplitudes with increasing lubricant amounts (Fig. 4).

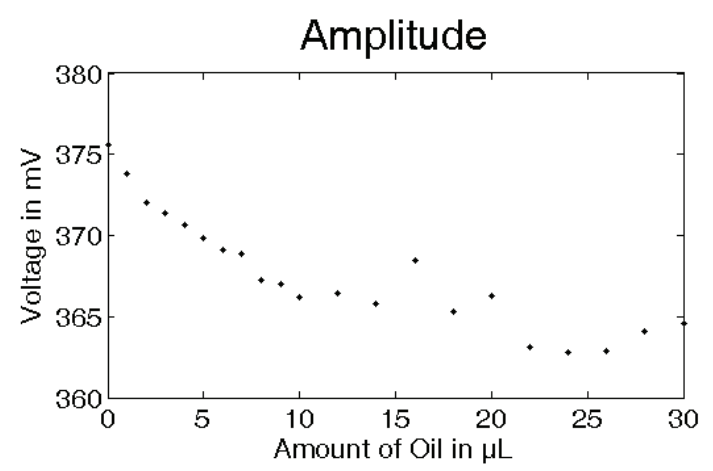

Transmission time

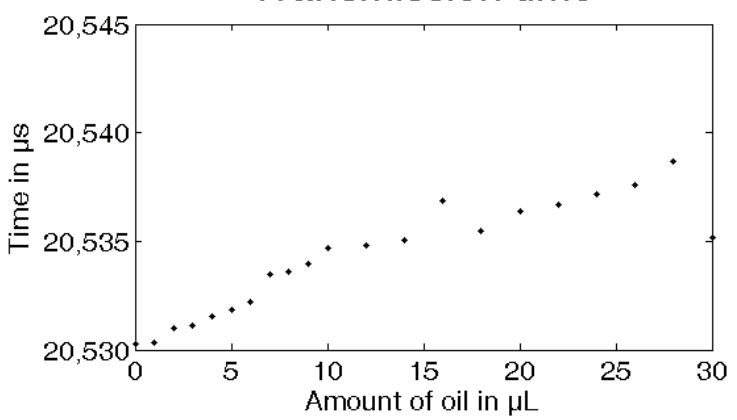

Standard deviation of the amplitude

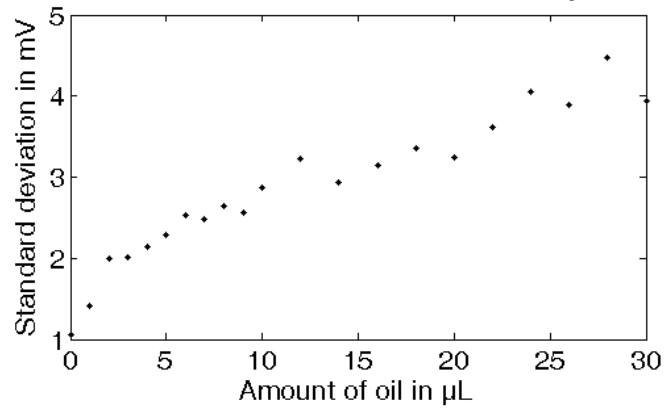

Standard deviation of the transmission time

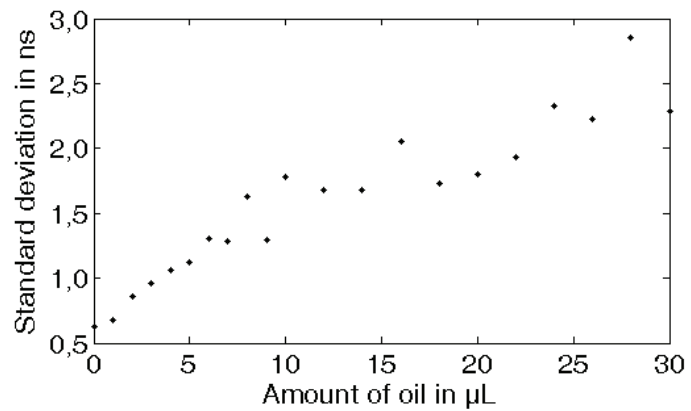

Fig 4: Changes of baseline values of the signal parameters and standard deviations of signal parameters during one measurement run measured at the cylinder roller bearing of type N306 - E - TVP2 with oil lubricant at a rotational speed of $360 \mathrm{rpm}$.

Corresponding measurements with grease lubricant at a ball roller bearing revealed significant differences, in particular with respect to the signal amplitude, which no longer depended monotonously from the lubricant amount (Fig 5).

\subsection{Rotation of rolling elements}

The frequency of the modulation of the signal parameters turned out to be strongly correlated with the rotational speed of the bearing (Fig. 6). Assuming that this modulation is caused by each rolling element at each passage of the emitter transducer, a relationship between the modulation frequency and the rotational frequency of the bearing can be established according to formula (1):

$$
\mathbf{f}_{\text {modulation }}=\frac{\mathbf{N}_{\text {rolling elements }}}{2} * \mathbf{f}_{\text {bearing }}
$$




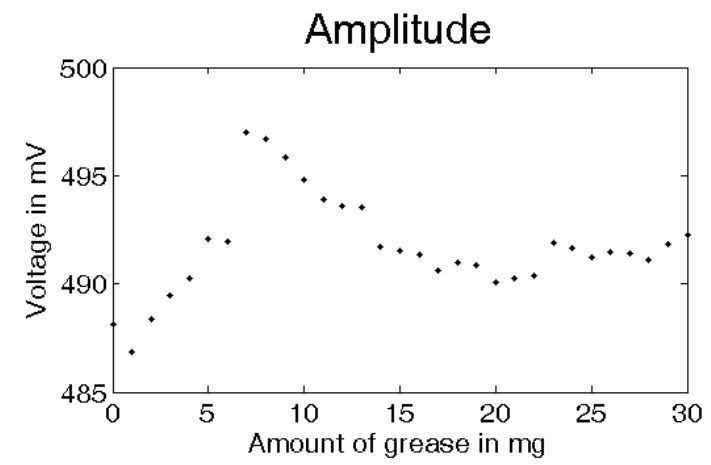

Standard deviation of the amplitude
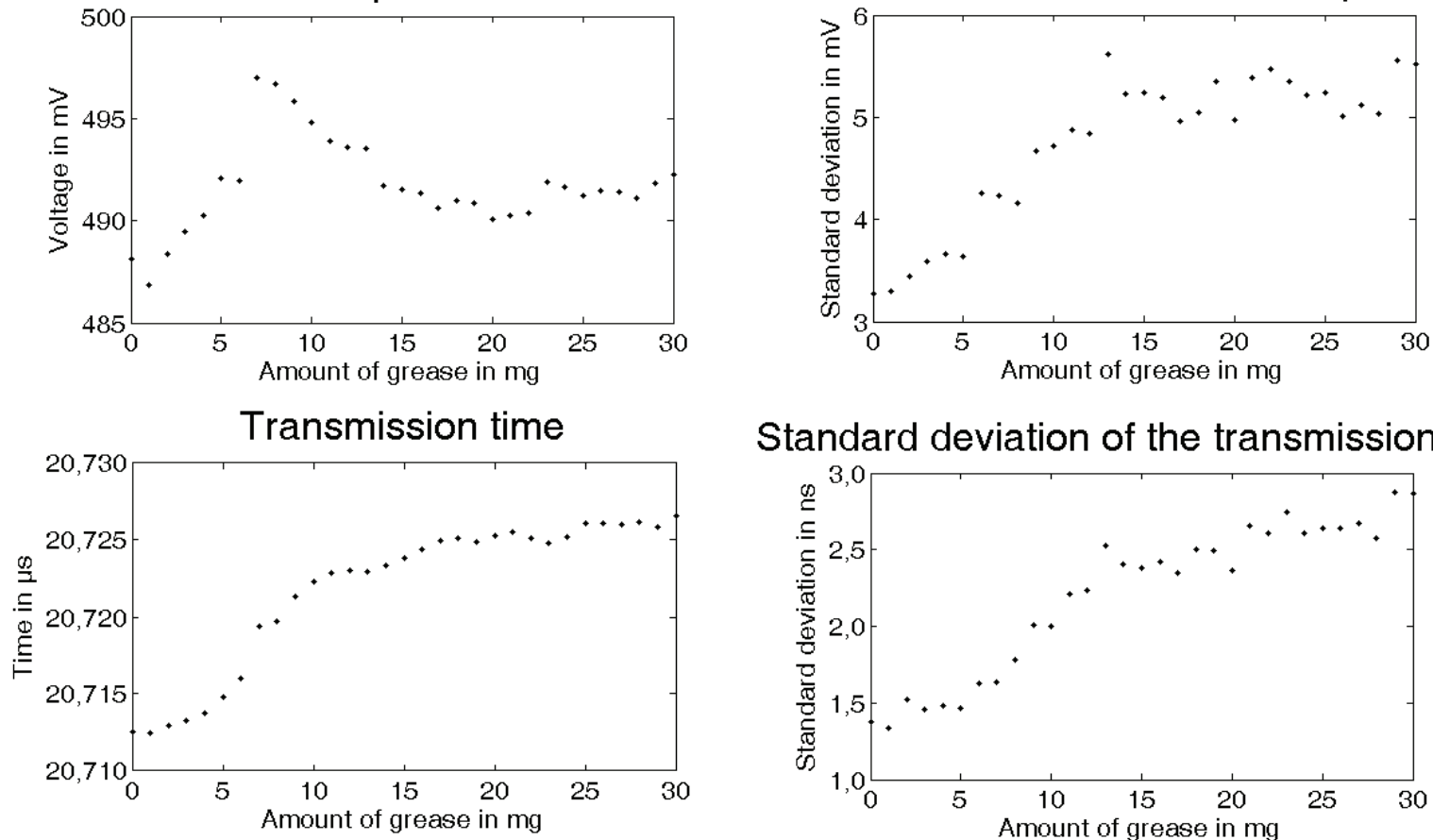

Standard deviation of the transmission time

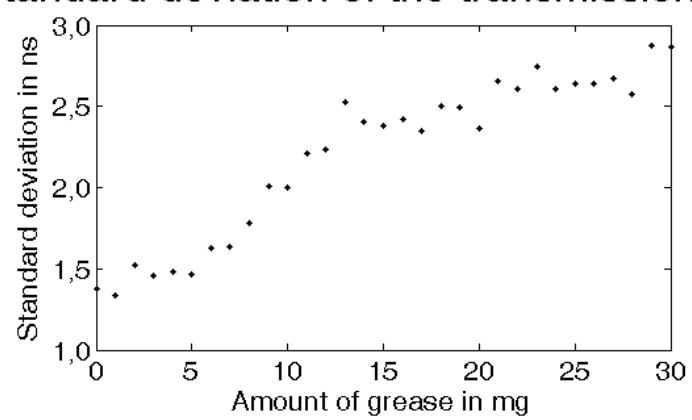

Fig. 5: Changes of baseline values of the signal parameters and standard deviations of signal parameters during one measurement run measured at the ball roller bearing of type 6306 with grease lubricant at a rotational speed of $360 \mathrm{rpm}$.

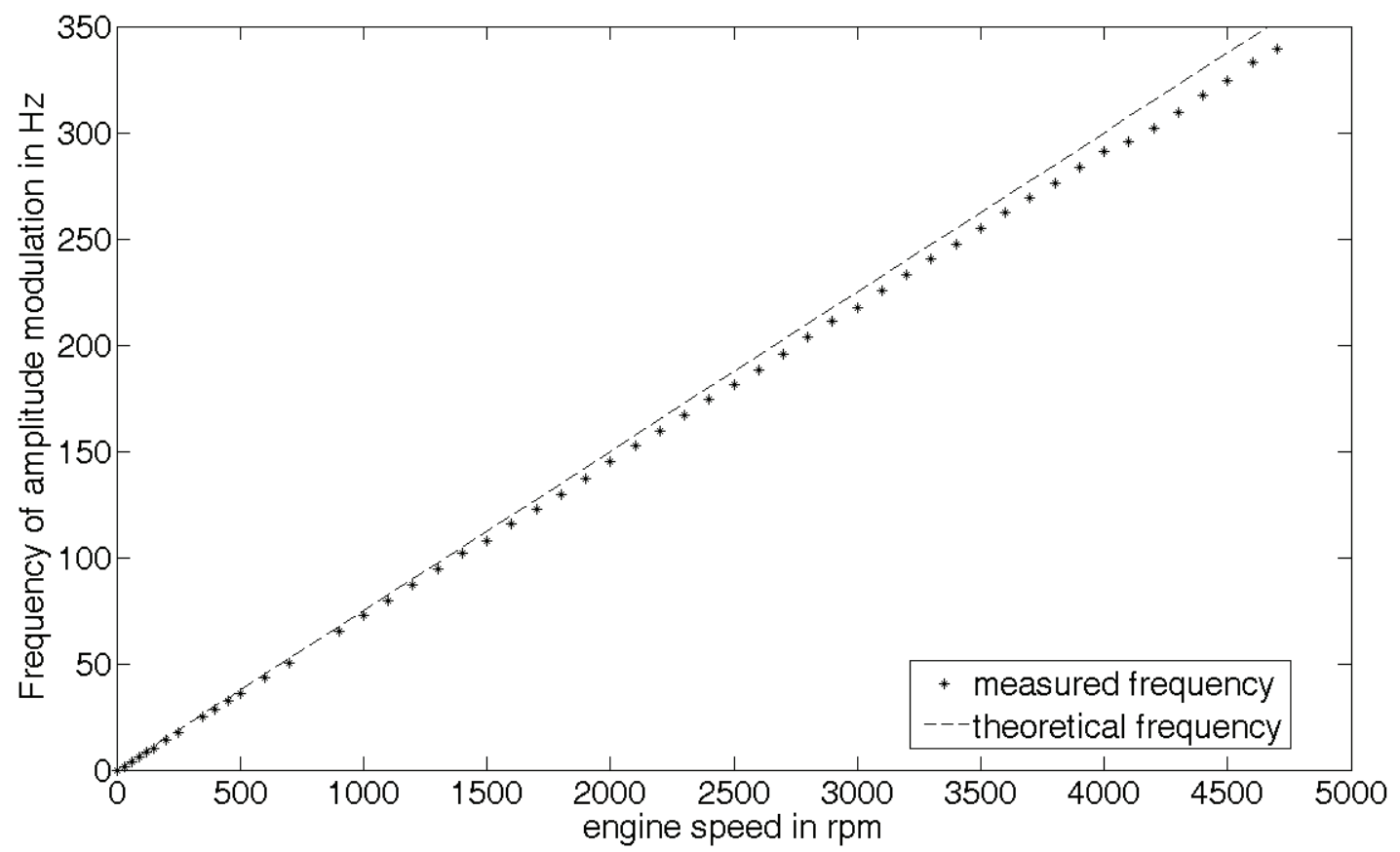

Fig. 6: Measured frequency of the amplitude modulation in comparison with calculated values as function of the rotational speed of the bearing. 
In fig. 6 the calculated values of the modulation frequency are compared with the measured results; a nice correlation has been observed, but also a significant deviation between the calculated and the measured curves, which has been attributed to a slip of the cage containing the roller elements with respect to the inner ring of the bearing.

\section{Discussion and conclusion}

The periodic modulation of signal parameters results from the movement of the rolling elements with respect to the fixed emitter transducer: In the rotating bearing the conditions for mode conversion near the emitter transducer at the inner surface of the outer shell of the bearing are changing periodically during the passage of rolling elements. In the interstice between the rolling elements the lubricant may accumulate and may cause acoustic leakage due to the mode conversion effect, whereas in the contact area with the rolling elements there may be some acoustic crosstalk with the surface of the rolling elements, but with different influence on the signal parameters compared with the acoustic leakage into the lubricant. The regular arrangement of rolling elements explains the period modulation pattern in the rotating bearing, whereas acoustic leakage into the lubricant is considered to be responsible for the increased changes of the signal parameters with increasing lubricant amount in the bearing. The nonmonotonous changes of the signal amplitudes with lubricant amount in the case of grease, on the other hand, thus may be attributed to a non-homogeneous distribution of grease in the interstice between the rolling elements, which may influence the mode conversion in an irregular way.

Another result of potential interest for future applications is the observation of a slip of the rolling elements and correspondingly of the moving cage containing those elements, in particular with respect to the effect of an external loading of the bearing. Therefore the investigations will be extended to different loads on the bearings in future.

\section{Acknowledgements}

The authors gratefully acknowledge the funding of this research by Bundesministerium für Bildung und Forschung (BMBF) with the program "FHprofUnt" and helpful support from colleagues of the Institute of Sensor and Actuator Technology. One of the bearings used in these investigations was supplied by FTE automotive $\mathrm{GmbH}$. The Institute of Sensor and Actuator Technology is supported by European Union within the program "EFRE" (project "Sensolutions").

\section{Literature}

[1] B. W. Drinkwater and R. S. Dwyer-Joyer, "The on-line measurement of lubricant film thickness for condition monitoring," Non-Destr. Test. Cond. Monit., vol. 46, pp. 456-460, 2004.

[2] N. Jamuludin, D. Mba and R. H. Bannister, "Monitoring the lubricant condition in a low-speed rolling element bearing using high frequency stress waves”, J. Process. Mech. Eng., vol. 216, pp. 73-88, 2002.

[3] G. Lindner, M. Schmitt, J. Schubert, S. Krempel and H. Faustmann, „On-line surveillance of lubricants in bearings by means of surface acoustic waves”, IEEE Trans. Ultrason. Ferroelectr. Freq. Control, vol. 57, no. 1 , pp. $126-132,2010$.

[4] F. Herrmann, B. Jakoby, J. Rabe and S. Bütttgenbach, „Microacoustic sensors for liquid monitoring“, Sensors Update, vol. 9, no. 1, pp. 105-160, 2001.

[5] G. Lindner, "Sensors and actuators based on surface acoustic waves propagating along solid-liquid interfaces”, J. Phys. D., vol. 41, art. No. 123002, 2008. 